\title{
Les relatives en français vernaculaire
}

\author{
Pierre Larrivée $^{1}$ et Marie Skrovec ${ }^{2}$ \\ ${ }^{1}$ Centre de Recherche Inter-langues sur la Signification en COntexte (CRISCO) - EA 4255 - Université \\ Caen Normandie (France) \\ ${ }^{2}$ Laboratoire Ligérien de Linguistique (LLL) - UMR7270 CNRS - Université d’Orléans (France) \\ pierre.larrivee@unicaen.fr, marie.skrovec@univ-orleans.fr
}

Résumé. L'étude fondatrice de Keenan and Comrie (1977) ordonne les fonctions grammaticales du pronom relatif sur une échelle d'accessibilité (sujet, objet direct, indirect, génitif, etc.). On s'attend ainsi à ce qu'un pronom relatif en fonction plus accessible soit plus fréquent, moins sujet à l'erreur de performance et moins soumis à des collocations. L'objectif de ce travail est d'évaluer les prédictions de la hiérarchie d'accessibilité en examinant le comportement des relatives en français vernaculaire. Pour ce faire, les manifestations des relatives sont analysées dans un sous-corpus d'environ 900000 mots d'ESLO2. L'analyse montre que plus il est accessible, plus un relatif est fréquent, et moins il est sujet à l'erreur de performance. Le travail suggère en outre qu'un relatif moins accessible entre plus régulièrement dans des schémas collocatifs, bien que les schémas attestés n'ont pas la répartition informationnelle quasi-catégorique qu'attestent les données vernaculaires de Duffield et Michaelis (2011) pour l'anglais, ce qui exclut une analyse uniquement constructionnelle pour le français. On contribue donc à (in)valider des hypothèses sur les causes de la hiérarchie d'accessibilité.

\begin{abstract}
The foundational work by Keenan and Comrie (1977) is proposing to rank syntactic functions of relative pronouns on an accessibility hierarchy (subject, direct object, indirect object, genitive, and so on). It predicts that a more accessible relative pronoun is more frequent, less liable to performance errors and less bound to collocations. The purpose of this paper is to evaluate these predictions. It examines the behaviour of relatives in contemporary vernacular French, using a POS-tagged sample of ESLO2 corpus. Analysis of the data shows that a more accessible relative pronoun is indeed more frequent and less liable to performance errors. Also, a less accessible relative pronoun is more regularly involved in collocations, although the attested collocations in French do not relate to information structure in the quasicategorical way they do in English (Duffield et Michaelis 2011), thus excluding a constructional analysis for French. The paper thus makes a contribution to the assessment of the causes of the accessibility hierarchy.
\end{abstract}




\section{Introduction}

Les études sur la proposition relative (inter alii Alexiadou et al 2000, Kidd 2011, Hendery 2012) lui attribuent généralement trois caractéristiques :

- c'est une proposition subordonnée qui modifie un nom (par exemple Diessel et Tomasello 2000)

- introduite par un pronom qui réfère à un antécédent (notamment Haspelmath 2010)

- qui restreint le potentiel référentiel du groupe nominal concerné (entre autres Comrie et Kuteva 2005)

Or, chacune de ces caractéristiques est discutable. C’est ce que montrent les relatives sans antécédent (Pierrard 1988) du type Qui dort dîne : elles ne modifient pas un nom, ne réfèrent pas (nécessairement) à un antécédent et donc n'en restreignent pas l'extension. C'est la raison pour laquelle nous préférons nous référer à une définition syntaxique de la relative. Syntaxiquement, la relative est une subordonnée dont le marqueur d'intégration a une fonction dans la proposition qu'il introduit. Cela le distingue de la complétive dont le subordonnant n'a pas de fonction au sens classique dans cette proposition. On peut ainsi distinguer les deux séquences suivantes :

L’idée qu'elle considère est curieuse.

L’idée qu'elle considère sa candidature est curieuse.

Dans le premier exemple, le pronom que a la fonction d'objet dans la proposition régie (il s'agit de considérer une idée), contrairement au deuxième exemple où que est un connecteur sans fonction d'objet (il est question de considérer une candidature). On en conclut que (1) contient une relative là où (2) comprend une conjonctive. Cette définition permet également de, reconnaitre que la relative restrictive et la relative non-restrictive relèvent du même schéma syntaxique, comme les relatives avec ou sans antécédent.

Le but de ce travail est de rendre compte du comportement des propositions relatives en français vernaculaire. Par vernaculaire, nous entendons les pratiques non-préparées et non-surveillées d'une langue. Nous supposons que la spontanéité de ces pratiques révèle la compétence immédiate des locuteurs et que cette compétence est davantage susceptible de révéler les principes et régularités qui sous-tendent l'organisation des langues que les pratiques normées qui relèvent d'un apprentissage ultérieur (voir en particulier Meisel, Elsig et Bonnesen 2011). Nous nous concentrons ici sur le français de France, la question de savoir si le vernaculaire de cette variété diverge de celui d'autres communautés restant soumise à une résolution empirique (pour le français québécois, voir par exemple, Lefebvre et Fournier 1978 ; pour la diachronie du français en rapport avec certaines de ses variétés, voir Auger 1995). Un domaine pour lequel la distinction entre pratiques vernaculaire et normée est particulièrement sensible pour le français de France est en effet celui des propositions relatives. Cela est illustré par la fréquence et la distribution de marqueurs comme dont et lequel comme le notent Claire Blanche-Benveniste et Philippe Martin :

Les difficultés que rencontrent les maîtres pour en enseigner l'usage à l'école primaire montrent bien que ces formes ne sont pas intégrées dans la connaissance première que les enfants ont de la grammaire. Ce sont des formes fréquentes dans le langage littéraire, dans le langage juridique et bureaucratique et dans certaines routines professionnelles, mais la plupart des locuteurs les utilisent peu dans leurs conversations et seulement dans des tournures formulaires. (Blanche-Benveniste et Martin 2010 : 101)

Il s'agit donc à partir d'une analyse de corpus de productions orales vernaculaires du français de France, de rendre compte de l'usage effectif des relatives. Nous présentons d'abord le débat sur la nature des contraintes déterminant le comportement général des relatives. Ce débat lancé par Keenan et Comrie 
(1977) concerne le rôle respectif du traitement des séquences et de l'organisation constructionnelle (Michaelis et Duffield 2015) : est-ce parce qu'elles sont plus faciles à traiter ou parce qu'elles sont structurées par des constructions spécifiques que les relatives «simples " sont moins contraintes que les relatives « complexes »? En admettant qu'elles s’y manifestent, de quelle façon ces contraintes s'expriment-elles dans la grammaire du français ? Après la description de la méthode de l'étude, nous procéderons à la description des conditions syntaxiques et interprétatives associées à l'usage de chacun des subordonnants pertinents dans le corpus choisi. Nous conclurons enfin en évaluant la convergence entre les conditions d'usage des relatives en français vernaculaire et les prédictions formulées dans les débats actuels sur la cause des contraintes dans le comportement des relatives à travers les langues.

\section{Arrière-plan théorique}

Les propositions relatives sont généralement envisagées en termes de complexité. L’idée est que l'intégration de deux propositions par le biais d'un item qui a un rôle syntaxique dans la proposition qu'il introduit rend la relative plus complexe que d'autres types de relations. On s'attend donc à ce que les structures plus «simples» soient moins l'objet d'erreurs de performance, soient acquises plus précocement, et soient plus fréquentes dans une langue et à travers les langues que les structures plus « complexes ».

C’est ce que reflète l'étude fondatrice de Keenan and Comrie (1977). Ces auteurs proposent une hiérarchie d'accessibilité s’appliquant aux pronoms relatifs.

Accessibility Hierarchy :

$$
\mathrm{SU}>\mathrm{DO}>\mathrm{IO}>\mathrm{OBL}>\mathrm{GEN}>\mathrm{OCOMP}
$$

Cette hiérarchie implique que le pronom relatif sujet est plus « accessible » que l'objet, l'objet que l'objet indirect, l'objet indirect que l'instrument, l'instrument que le génitif, et le génitif que le comparatif. Selon la perspective typologique qui intéresse les auteurs, toutes les langues auront un pronom relatif sujet, et on présume qu'une langue permettant une relativisation génitive permettra celle objet indirect, mais que l'inverse n'est pas nécessairement vrai. D’après cette hiérarchie, nous formulons l'hypothèse qu'un pronom relatif sujet sera plus fréquent dans une langue qu'une pronominalisation objet indirect, qui sera elle-même plus fréquente qu'une pronominalisation génitive.

Si une telle hiérarchie existe, elle devrait se refléter dans l'acquisition des langues, qu'elles soient maternelle ou seconde. La complexité semble jouer un rôle certain dans la progression de l'acquisition des relatives :

The earliest relative clauses we found in the data occur in topicalization constructions that are only a little different from simple sentences: they contain a single proposition, express the actor prior to other participants, assert new information and often occur with main-clause word order. In the course of the development, more complex relative constructions emerge, in which the relative clause is embedded in a fully-fledged main clause. We argue that German relative clauses develop in an incremental fashion from simple non-embedded sentences that gradually evolve into complex sentence constructions. (Diessel et Tomasello 2000)

La hiérarchie elle-même n’est néanmoins pas universellement avérée par les études acquisitionnelles. Alors qu'une bonne partie des études théoriques sur l'acquisition se concentrent surtout sur la question de savoir si la relative implique ou non un mouvement syntaxique (par exemple Guasti et Schlonsky 1995), les travaux plus empiriques mettent en avant des résultats contrariant la hiérarchie d'accessibilité. Un article ancien de Hawkins (1989) propose que l'acquisition des relatives en français se fonde sur des 
informations de linéarité plus que de fonction. La publication de Guasti et collaborateurs (2012) montre que des informations morphologiques fournies par les relatives du grec influencent leur taux d'acquisition. Les langues d'Extrême-Orient sans mouvement du relatif (mandarin, japonais, coréen) contreviennent également aux prédictions de la hiérarchie (voir certaines des contributions à Kidd 2011 par exemple) :

With respect to relative clause (RC) production, children in different languages have been found to produce more subject relative clauses earlier than object relative clauses (Keenan \& Comrie 1977). However, predictions based on the NPAH were not borne out in some other studies, which have posed challenges to NPAH. For example, when examining relative clause production in Cantonese children, two studies (Matthews \& Yip 2002, Yip \& Matthews 2007) found that Cantonese-English bilingual children produced Cantonese object RCs earlier than subject RCs. The reason might be due to the fact that Cantonese object relative clauses actually have a SVO word order, which is similar to the canonical SVO word order in Cantonese. (Lin 2015)

Malgré les objections provenant de ces études, on peut supposer que la hiérarchie d’accessibilité demeure un principe opérant : comme le notent Behney et Gass, il existe « a clear preference in production and learning order that reflects the A(ccessiblity) H(ierarchy) » (2013 : 56). Quelle est la motivation de ce principe ? C’est le débat que reprennent Michaelis et Duffield (2015). L'explication générative de la prépondérance des subordonnées relatives introduites par un pronom sujet (ce que nous appellerons relatifs sujets) recourt à la facilité de traitement d'un pronom proche de la trace que laisse son déplacement. La distance entre le pronom relatif sujet à l'initiale de la proposition et la trace qu'il laisse en position de sujet de cette proposition est moindre et les deux entités sont séparées par moins de projections que ce n'est le cas avec l'objet direct par exemple.

(3) L’idée qui _ est considérée est curieuse.

$$
\text { L’idée qu'elle considère _ est curieuse. }
$$

La distance typique en nombre de mots et en nombres de projections rendrait donc le relatif sujet plus facile à traiter. Michaelis et Duffield (2015) font cependant valoir que la prépondérance des relatifs sujets ne se manifeste pas également dans tous les contextes. Ils examinent un corpus d'anglais américain vernaculaire étiqueté syntaxiquement, le Switchboard Treebank corpus, selon la fonction du relatif dans la proposition et selon la fonction du terme que la proposition relative détermine. Les 2640 occurrences extraites confirment la prépondérance des pronoms relatifs sujets, qui représentent les deux-tiers des occurrences totales. Cette prépondérance ne se manifeste pas dans tous les contextes cependant : les relatifs sujets ne sont dominants que quand le SN qu'ils déterminent est un complément direct ou indirect ; quand ce SN est un sujet, c'est un relatif objet (in)direct qui représente 60\% des occurrences de relatifs. En outre, les relatifs sujets sont plus susceptibles de modifier un nom indéfini. Il va sans dire que cette observation contredit une analyse structurale, puisqu'une grammaire contextuellement libre n'a pas de raison de distinguer la distance entre pronom et trace selon la fonction de l'antécédent. Le constat va également à l'encontre de l'explication esquissée par Diessel (2007) selon qui la prépondérance des relatifs sujets serait due à l'aspect agentif de la fonction. Les auteurs avancent que la cause des comportements tient à des routines constructionnelles: la structure 'nom indéfini + relative' sert à introduire une information nouvelle, ce que ne fait typiquement pas le sujet (sur la difficulté avérée des indéfinis en sujet antéposé à cause du conflit entre information nouvelle supposée par l'indéfini et information ancienne supposée par le sujet, voir par exemple Cappeau et Deulofeu 2001). 
Sur cet arrière-fond théorique, on peut donc se demander à propos des relatives en français vernaculaire :

- si elles suivent la hiérarchie d’accessibilité, et si en particulier les relatifs sujets sont plus fréquents et moins affectées par l'erreur que les autres ;

- si cette fréquence se manifeste dans toutes les configurations syntaxiques, ou si elle est liée à des configurations d'usage particulières.

La façon dont cette étude répond à ces questions est décrite dans la section qui suit.

\section{Méthode}

L’objectif de ce travail est d'évaluer le comportement des relatives en français vernaculaire, au regard en particulier de la notion de complexité. Pour ce faire, il s’attache à la description du phénomène dans le corpus ESLO 2. Corpus oral constitué depuis 2008 par un collectif de linguistes du LLL (Eshkol et al. 2012) et deuxième volet d'une collecte réalisée par des chercheurs britanniques entre 1968 et 1971, les Enquêtes Sociolinguistiques à Orléans 2 regroupent un large éventail d’interactions orales reflétant les pratiques langagières des habitants de la ville. Composée selon des modes de collecte diversifiés qui vont de l'entretien sociolinguistique à la saisie d'interactions non élicitées et en l'absence du chercheur, en passant par l'observation participante, cette archive contient des productions différenciées du point de vue de leur degré de spontanéité et de formalité notamment (cf. Baude et Guérin à paraitre). Nous avons utilisé un sous-corpus d'environ 900000 mots représentatif de différents genres ${ }^{1}$ qui a été étiqueté grâce au logiciel Treetagger pour l'oral (Benzitoun, Fort et Sagot 2012). Nous nous sommes concentrés sur les échanges «Diachronie » (entretiens auprès de locuteurs âgés déjà enregistrés dans ESLO1), « Entretiens Jeunes » et sur le module "Repas », dont les enregistrements allient haut degré de familiarité entre locuteurs, absence de préparation thématique, forte interactivité et faible degré de formalité. Les pronoms relatifs sont extraits des échanges avec leurs contextes gauche et droit. Chaque occurrence est annotée selon la nature de l'antécédent (identité, catégorie nom ou pronom), la fonction du relatif dans la proposition (sujet, objet, attribut, objet indirect, autre complément), et la fonction du groupe constitué par l'antécédent et la relative dans la proposition dominante (sujet, objet, attribut, objet indirect, autre complément). Comme maints autres auteurs, nous sommes attentifs à la participation des marqueurs à des structures clivées, afin d'évaluer le taux éventuel d'intégration à ces constructions. Les clivées forment une famille potentiellement large dont nous retenons les clivées en c'est (Nølke 1983, Collins 1991, Scappini 2006) et celles en il y a (Lambrecht 1987, 1988, 2001, Morel 1992, Matras et Sasse 1995, Méry 2011), sans pourtant inclure les structures moins fréquentes de type j'ai (ma mère qui flippe). Nous notons enfin les structures tronquées, les corrections et les répétitions, ce qui nous permet d'évaluer la difficulté supposée croissante des marqueurs plus complexes. Ces annotations nous permettent d’établir la tension entre productivité et figement au regard du débat entre traitement et constructionnalité.

\section{Description des relatives par subordonnant}

\subsection{Description de qui}

Qui est de loin le relatif le plus fréquent dans le corpus. Il représente 7623 occurrences brutes. Les échanges « Diachronie », « Entretiens Jeunes » et « Repas » représentent respectivement 827, 1165 et 643

${ }^{1}$ repas en famille et entre amis, interaction de commerce, micro-trottoir (sortie de cinéma, demande d'itinéraire), suivi d'un locuteur tout au long de ses activités quotidiennes, entretiens avec un large panel de locuteurs (dont des témoins déjà enregistrés pour ESLO1), émissions de radio locale, conférences universitaires, assemblées générales, discours. 
attestations brutes. Ce sont les 200 premiers cas bruts de chaque échange qui ont été retenus pour le codage. L'étiquetage syntaxique est relativement satisfaisant, et n’a entrainé l'exclusion que de 35 exemples qui relevaient de l'interrogation directe ou indirecte, le codage concernant un total de 565 occurrences. Elles incluent deux emplois qui représentent une fusion de que et d'un il impersonnel

$$
\begin{aligned}
& \text { a. } \quad \text { y a tout tout ce qui faut à Paris } \\
& \text { b. } \quad \text { tout ce qui y a comme temps }
\end{aligned}
$$

Tous les cas de qui annotés ont un antécédent. Ce à quoi réfère le relatif se divise en $415 \mathrm{SN}$ et 148 pronoms, c'est-à-dire $74 \%$ et $26 \%$ des 563 occurrences ; les 2 occurrences manquantes étant dues à 2 troncations rendant l'antécédent non identifiable. Les pronoms incluent au premier chef les démonstratifs (55 ce, 4 ça, 10 celui, 1 celle, 10 ceux, 4 celles, pour un peu moins de 57 \% des pronoms et $15 \%$ du total), les pronoms "personnels » (3 moi, 5 toi, 10 lui, 13 en, 2 les clitiques), des indéfinis ( 7 quelqu'un, 5 quelque chose, 1 personne, 1 tout), des interrogatifs (2 quoi, 8 que, 7 qui).

Le relatif a une fonction dans la proposition qu'il introduit. Il est sujet, avec 557 cas des 563 occurrences. On relève 2 exemples de qui employés avec une préposition (objet indirect ou complément prépositionnel):

(5) a. que les sœurs euh à qui appartenait cette clinique euh voulaient euh non non c'est

b. $\quad y$ a les euh les \& ado euh véritables avec qui on travaille euh on travaille

une production atypique comme objet direct :

j'aime pas l'Anglais c'est ce qui vous disiez oui oui oui

et 3 instances de troncation rendant la fonction dans la subordonnée non identifiable. S'ajoutent à ce chiffre les 2 cas de fusion de que à il donnés ci-dessus.

Les fonctions assumées par le syntagme formé par le relatif et l'élément qu'il détermine sont diverses. Ce syntagme est tour à tour sujet (23), objet direct (278), attribut (148), objet indirect (12), circonstant (46), complément d'un adjectif (5) ou d’un nominal (12).

(7) a. il faut modérer ça parce que finalement euh les femmes qui ont él- ont élevé leurs enfants ont ont pas de problème de cet ordre là (ESLO2_DIA_003_C)

b. est c'est ouais euh moi j'ai des élèves qui euh qui étaient atteints de rétinite avec une surdité (ESLO2_DIA_003_C)

c. c'était une grande enfin bon c'est plus elle qui m’a adopté que moi (ESLO2_DIA_017_C)

d. qui sont marqués avec avec le sang de l'individu qui a été tué hm hm hm hm bon (ESLO2_DIA_003_C)

e. $\quad$ que conseillez vous de faire à Orléans à quelqu'un qui vient ? (ESLO2_DIA_017_C)

f. que les les sujets soient plus conscients de de ce qui peut l- vla- leur arriver (ESLO2_DIA_003_C)

31 cas sont autonomes (sans proposition régissante).

g. hop un morceau de beurre qui va fondre hm 
4 occurrences sont tronquées de façon à ce que la fonction du syntagme ne soit pas identifiable. Au total, la moitié des syntagmes sont objets directs, et il n’y a que 4 \% de syntagmes sujets en comptant les 12 des 17 dislocations en rapport avec cette fonction.

(8) a. en plus je je la dame qui me téléphonait là c'est la présidente

b. mais ce qui m'intéresse c'est les gens c'est les personnes

Qui participe d'une clivée à un taux significatif, dans 78 clivées en c'est, dans 4 semi-clivées, et dans 152 présentatives en (il) y $a^{2}$ - ces dernières se divisant en 87 instances à interprétation existentielle et 65 évènementielle (cf. typologie de Lambrecht 2000).

(9) a. $\quad \mathbf{y}$ a des gens qui parlent très bien le français et et qui savent ce que chaque mot veut dire (ESLO2_DIA_003_C)

b. $\quad$ oui y a eu énormément de choses qui ont changé (ESLO2_DIA_003_C)

La relative introduite par qui n’est pas exempte de problèmes de performance. On relève 63 répétitions, 17 troncations, 3 productions atypiques en (6)-(8), mais apparemment aucune hésitation sur le choix du relatif, ce qui place la troncation à $3 \%$ des occurrences.

Qui est ainsi un relatif fréquent, avec un antécédent surtout nominal, à fonction presque uniquement de sujet dans la proposition qu'il introduit, et une variété de fonctions dans la proposition qu'il introduit. Le taux de troncation reste faible à $3 \%$ des occurrences.

\subsection{Descriptions de que}

Que est le deuxième marqueur le plus fréquent après qui dans le corpus. Il compte 1199 occurrences brutes pour 'que' et 1173 occurrences brutes pour 'qu', de ces dernières cependant, on peut légitimement se demander si toutes les occurrences représentent bien le pronom objet, ou une contraction de qui : un examen des 100 premières occurrences de l'échange « Diachronie » donne 14 exemples dans ce cas.

(10) a. bien euh et p- euh là c'est une question qu' est peut-être superflue mais quand euh quand euh je vais

b. même y en a qui présentent le journal qu'en font hein

Ce sont les occurrences des échanges «Diachronie », «Entretiens Jeunes » et « Repas » qui ont été codées, représentant 72, 84 et 88 occurrences de relatives chacune (soit un nombre brut de 117, 144 et 155 respectivement dont il a fallu soustraire 45, 60 et 67 occurrences mal étiquetées, concernant des conjonctifs précédés d'un graphème 'ce' (dans parce que notamment). Les 244 occurrences retenues contiennent différents cas de troncation, que nous commentons ci-dessous.

Toutes les occurrences de que ont un antécédent. Il s’agit de façon massive du pronom ce, pour 212 exemples, auquel s’ajoutent les démonstratifs celui (3) et ceux (4), ceux-là (1), celle (4) et celles (3); on compte en outre 1 exemple avec autre chose et 1 autre avec quelque chose. Seules 6 occurrences proposent un groupe nominal comme antécédent. (Les 9 occurrences manquantes par rapport au total de 244 s'expliquant par des cas d'hésitation et de troncation qui rendent incertaine l'identification du

\footnotetext{
${ }^{2}$ Pour des raisons de temps, les variantes personnelles (j'ai $X$ qui) et à verbe de perception n'ont pu être
} prises en compte. 
référent.) Au total, $90 \%$ des exemples ont pour antécédent ce, $97 \%$ un démonstratif, et 97 \% un pronom, avec moins de 3\% de SN. Cela confirme l'idée que l'antécédent du relatif en français vernaculaire a « une charge lexicale faible ou nulle » (Blanche-Benveniste et Martin 2010 : 99, qui mentionne quelqu'un qui, là où), qui est cependant contredite par le cas de qui avec 15\% d'antécédents démonstratifs et $73 \%$ d'antécédents SN. Selon nos observations, c'est donc l'antécédent du relatif objet qui revêt une charge lexicale faible, tandis que l'antécédent du relatif sujet est davantage lexical.

La fonction des occurrences de que dans la proposition relative est très majoritairement objet direct (194), à quoi s'ajoutent 21 attributs. Des emplois atypiques sont relevés. D'une part, 3 objets directs sont exprimés sans la préposition attendue avec le verbe dépend.

tout dépend ce que l'on regarde bon bah

Dans 3 autres cas concernant ‘que’, il y a hésitation sur le relatif

(12) a. sur un terrain d- de de terre avec des bosses que qui sont faites par euh par eux

b. oui alors là ça a été l'éjection euh dont que j'ai fait partie hein il a fallu que je

c. des fois y en a que y en a qui utilise un j'aime pas quand

les décalages avec le nombre d'antécédents s’expliquant par les 23 cas de troncations où la proposition non terminée ne permet pas de repérer la fonction du relatif. 90 \% des exemples avec fonction dans la relative comprennent donc un objet direct, et $100 \%$ un complément direct (objet direct ou attribut).

C’est l'antécédent de que que les propositions relatives déterminent. Le groupe formé par l'antécédent et la relative complément (218 occurrences) a des fonctions diverses dans la proposition dominante. Ces propositions ont fonction d'objet direct (98), d'attribut (44), d'objet indirect (21), de groupe comparatif (8), de complément prépositionnel (17), 2 circonstants et 16 dislocations (10 à gauche et 6 à droite).

(13) a. $\quad$ il est de toute façon il n'écoute pas ce que tu dis (ESLO2_REPAS_1254_C)

b. $\quad$ C'est ce que j'allais te dire (ESLO2_REPAS_1257_C)

c. Assieds-toi on comprend rien à ce que tu racontes (ESLO2_REPAS_1257_C)

d. à transporter euh sur internet hein oui bah comme celui que j'ai porté la dernière fois là euh la petite (ESLO2_REPAS_1259_C)

e. $\quad$ non mais sauf ceux que tu as vus toi (ESLO2_REPAS_1253_C)

f. tu peux mettre des petites herbes aussi aromatiques hm selon ce tu aimes ou pas histoire d'aromatiser un peu

g. Vous avez adopté des enfants ils sont grands maintenant ce- celle que vous avez ramenée du Rwanda elle a quel âge maintenant (ESLO2_DIA_017_C)

On notera qu'aucune relative en que ne se rapporte à un sujet, ce qui va dans le sens d'une spécialisation fonctionnelle du relatif, un peu comme l’ont observé Michaelis et Duffield (2015), chez qui néanmoins c'est avec le sujet de la proposition dominante que le relatif objet est dominant On retrouve parmi ces exemples, 31 clivées et 16 semi-clivées, et 1 cas correspond à une dislocation à gauche. 12 exemples sont autonomes (sans proposition dominante). S’ajoutent 25 troncations et 1 hésitation. Les troncations représentent près de $11 \%$ des occurrences. 
Un emploi de que qui a été remarqué dans de nombreuses études sur le français vernaculaire est celui du « décumul » du relatif (Gadet 1995, Blanche-Benveniste et Martin 2010 : 98). Il s'agit d'un que marquant la subordination accompagné d'une expression anaphorique dans la relative marquant la nature du rapport fonctionnel en cause : un exemple en est la personne que je t'en ai parlé, plutôt que l'attendu la personne dont je t'ai parlé. Nous notons qu'il n’y a aucune occurrence d'un tel décumul dans les occurrences dépouillées, ni pour le sujet pour lequel la structure serait la plus importante selon Blanche-Benveniste et Martin (2010 : 98) ni pour une autre fonction.

En résumé, que est bien représenté, bien que nettement moins que qui dans le corpus. Son antécédent est à 97 \% un démonstratif, et il est exclusivement objet dans la proposition qu'il introduit. Le syntagme formé par la relative et son antécédent ne se rapporte jamais à un sujet. Le taux de troncation s’élève à $11 \%$ des occurrences.

\subsection{Descriptions de où}

Le relatif où est le troisième marqueur le plus fréquent, avec 841 occurrences. Dans les échanges « Diachronie », « Entretiens Jeunes » et « Repas » qui ont été codées, on retrouve 175 exemples.

Contrairement aux autres relatifs, où n’a pas toujours un antécédent, qui est absent dans 8 cas.
a. mais toi tu te mets où est Soren ?
b. où tu vois une personne connectée tu te dis tiens ça

La pro-forme là représente 9 autres cas. La très grande majorité des occurrences ont un SN comme antécédent (158, dont 14 en schéma collocatif avec moment).

(15) a. je pense que en particulier y a eu un moment où on a lé- lé- celui qui avait son bac il

b. le mercredi ouais généralement c'est là où y a le plus de monde

La fonction du relatif par rapport à la proposition qu'il introduit est celle d’un complément. Dans 5 cas, où est un objet indirect requis par la sous-catégorisation du verbe et a une valeur locative.

(16) a. c'était le truc où on allait beaucoup euh

b. dans le quartier où j'habitais avant

Les autres cas de complément diffèrent selon la valeur qu'ils assument. Il peut s'agir d’évoquer le lieu (55), le temps (49), ou de façon moins attendue une situation par rapport à une abstraction (51) ; ainsi, dans ce qui suit, on ne peut guère arguer sans risque de s'appuyer sur une métaphore abusive qu'une discipline ou qu'un métier est un lieu proprement dit.

(17) a. voilà oui hm euh j'enseigne dans une matière où y a beaucoup de préparation matérielle $\mathrm{hm}$

b. $\quad$ en plus qu'il y a quand même certains métiers où les femmes sont quand même plus plus performantes je crois

A cela s'ajoute 17 cas de troncation et 3 hésitations empêchant la détermination de la fonction du relatif dans la subordonnée qu'il introduit.

Le syntagme que contribue à former où avec son éventuel antécédent occupe des fonctions diverses. Sont concernés l’objet direct (46), l'attribut (43), d’objet indirect (9), des compléments prépositionnels (5), des 
compléments autres (38) et 3 cas correspondent à une dislocation gauche. Parmi ces fonctions, 39 représentent des clivées en c'est ou en il y a. Il n’y aucune fonction de sujet représentée. 20 séquences sont autonomes, sans proposition dominante. 10 troncations privent la relative de fonction identifiable dans la proposition dominante.

Le relatif où donne un taux significatif de troncations, avec 17 exemples, soit près de $10 \%$ des exemples des sous-corpus traités.

(18) a. on dit que c'est une ville où les gens où c'est froid et cetera euh moi je crois que

b. de toute façon tous les ans y a un moment où tu ah oui non mais euh là euh là c'

La fréquence de où est relativement faible.

\subsection{Descriptions de dont et des composés de lequel 3}

En appui à l’idée de la complexité des relatifs, dont et lequel sont réputés rares en français vernaculaire, comme le propose la citation de Blanche-Benveniste et Martin en introduction. Ils soulignent en outre qu'autant lequel semble complexe en relatif, autant ces difficultés ne se posent pas en interrogatif.

Ces assertions sont confirmées par les données des échanges «Diachronie », «Entretiens Jeunes » et « Repas ». Considérons d'abord le relatif dont. On compte 125 formes brutes dans le sous-corpus, et 19 dans les trois types d’échanges. Dans deux cas, il introduit une séquence averbale :

(19) a. les gens parlent leur langue et puis une ou deux autres dont l'Anglais

b. des cas de suicides dans dans certaines entreprises dont malheureusement la nôtre

Dans ces échanges, dont a toujours un antécédent, le plus souvent un SN (16), et 3 occurrences du pronom ce. Sa fonction dans la relative comprend 6 compléments d'un nom (dont 1 sujet), 9 objets indirects, 1 complément de manière, à quoi s'ajoute 1 cas atypique d’objet direct (ci-dessous (21)).

${ }^{3}$ Restées dans un premier temps non visibles car étiquetés automatiquement comme interrogatifs ou marqueurs du discours, 8 occurrences de quoi peuvent être relevées dans le corpus dépouillé. On relève une occurrence dans un complément oblique qui modifie un SN en position d'attribut :

DJ 39: bon euh alors que si c'est une musique à quoi faut prêter attention ça devient désagréable hein (ESLO2_DIA_003_C_10)

Les 7 autres occurrences de quoi dans le corpus dépouillé sont des relatifs indéfinis qui participent de la tournure figée avoir de quoi (personnelle ou impersonnelle comme ci-dessous):

ah bah vous avez de quoi lire là (ESLO2_DIA_048_C_20)

ça on a huit heures de philosophie par semaine $\mathbf{y}$ a de quoi avoir des migraines (ESLO2_ENTJEUN_08_C_1)

On trouve également 5 occurrences du segment lexicalisé n’importe quoi. 
La relative a fonction de complément du nom dans la proposition dominante. Hormis 3 séquences autonomes, le groupe formé par l'antécédent et la relative est objet direct (4), attribut (6), objet indirect (1), des compléments autres (5). Jamais la fonction sujet n’est concernée.

Un schéma collocatif bien connu est attesté par le corpus, c’est le rapport de dont avec le verbe parler, qui concerne ici 6 des 17 subordonnées conjuguées. 2 de ces occurrences avec parler sont introduites par une clivée.

Le taux de productions inattendues s’élève à 3 occurrences. Deux cas marquent une hésitation entre dont et que :

$$
\text { ah oui alors là ça a été l'éjection euh dont que j'ai fait partie hein }
$$

Un autre concerne une réalisation atypique comme objet direct du verbe subordonné :

parce que j'ai eu une femme dans mon atelier dont le ministre de l'époque est venu voir

La proportion de productions inattendues est donc de 17,6 \%, pour une forme employée avec le même verbe dans un tiers des cas, et dont le nombre total de réalisation est extrêmement faible.

On attend une situation comparable avec lequel. Les formes attestées dans l'ensemble du corpus sont des variantes de lequel (17 occurrences), auquel (11 occurrences) et duquel (1 occurrence). Les 94 formes brutes contiennent une bonne moitié d'occurrences interrogatives mal étiquetées. Les trois types d'échanges annotés donnent 7 formes seulement. Elles ont comme antécédent un SN, et jamais de proforme (et si en effet la séquence celle avec laquelle est acceptable, * ce avec lequel apparait agrammatical). Leur fonction dans la subordonnée est celle d'objet indirect pour les 2 auquel et de complément pour les 5 autres formes introduites par les prépositions sur et dans. L'antécédent nominal des relatives est objet (3) ou attribut (4), jamais sujet. 1 cas d'hésitation sur le relatif entre laquelle et dans laquelle est attesté :

(22) entre temps je mets là une grande poêle sur le feu histoire de la chauffer un peu laquelle dans laquelle d'accord je mets un peu de beurre

Cependant, on ne relève aucune troncation.

Les principaux résultats empiriques et leur signification pour les débats actuels sont résumés dans la section qui suit.

\section{Interprétation des résultats}

Cette étude décrit le comportement des subordonnées relatives en français vernaculaire sous le rapport de leur fréquence, de la nature de l'antécédent du relatif, de la fonction du relatif dans la proposition qu’il introduit, de la fonction du groupe constitué par la relative et ce qu'elle modifie, et de la présence de productions inattendues. La description contribue au débat sur la complexité des relatifs dont l'origine est pour certains la difficulté de traitement des relatifs plus complexes, et pour d'autres des schémas constructionnels spécifiques propres à tel pronom relatif. Cette discussion conclusive résume la façon dont les données informent les deux questions structurant l'étude :

- celle de savoir si les relatifs suivent la hiérarchie d’accessibilité, et si en particulier les relatifs sujets sont plus fréquents et moins sujets à l'erreur que les autres ;

- et si cette fréquence se manifeste dans toutes les configurations syntaxiques, ou si elle est liée à des configurations d'usage particulières. 
La hiérarchie d’accessibilité de Keenan et Comrie (1977) semble respectée : en particulier, le relatif sujet à 2635 formes brutes pour les trois types d’échanges considérés est plus fréquent que l'objet direct à 244 occurrences dans ces échanges, qui est plus fréquent que les objets «indirect » où (175), dont (19) et lequel (7). Qui représente ainsi près de $86 \%$ des formes. Il est de plus moins sujet à l'erreur de performance, avec 3\% de troncation, qui montent à $11 \%$ pour que, un peu moins de $10 \%$ pour où, et aucune pour dont et lequel, vraisemblablement à cause du faible nombre de productions dans les échanges (mais rappelons que dont présente un taux élevé de productions inattendues à près de $18 \%$ ). Même si la relation n'est probablement pas statistiquement significative du fait de la taille de l'échantillon, le taux d’erreur semble donc en rapport avec la hiérarchie d’accessibilité.

On observe par ailleurs que les fonctions des relatifs sont bien réparties entre eux, sujet pour qui, objet pour que, prépositionnel et circonstant en à pour où, prépositionnel en de pour dont et prépositionnel pour lequel. Un schéma collocatif qui se dégage est l'emploi des antécédents démonstratifs avec que, représentant $97 \%$ des occurrences. D’autres exemples sont les emplois de dont avec parler (35\% des occurrences) et de où avec moment (8\%). Une collocation transversale est donnée par la clivée, qu’il y a lieu de discuter. On dénombre 234 occurrences du relatif sujet qui impliquées dans une clivée (78 en c'est et 152 en il y a) ou une semi-clivée (4), ce qui représente 41,5 \% des cas annotés, tandis que pour le relatif objet que, on compte 49 clivées ( 9 avec SN, 21 avec la pro-forme ce 'c’est ce que’) et semi-clivées (18), ce qui correspond à une proportion de $20 \%$ des occurrences. Où compte 39 occurrences impliquées dans des clivées uniquement (22\% des cas). Enfin, dont et lequel (et ses composés), très peu nombreux on l'a vu, comptent respectivement 2 et 3 cas impliqués dans une clivée. En tout état de cause, l'annotation des relatifs permet d'appréhender les clivées, schémas constructionnels déjà décrits par ailleurs (BlancheBenveniste 2000, Lambrecht 2001), d'un point de vue quantitatif. On observe notamment que les clivées en il y a suivies d'un relatif sujet sont plus fréquentes que les clivées en c'est suivies d'un relatif sujet, contrairement à ce qui a été observé ailleurs (Blanche-Benveniste et Martin 2010). Nos analyses montrent également d'autres propriétés des clivées, notamment des préférences combinatoires qui définissent ces schémas constructionnels : ainsi, sur 152 clivées en il y a + SN (objet) impliquant un relatif sujet, 116 modifient un SN indéfini, tandis que sur 78 clivées en c'est + SN (attribut) impliquant un relatif sujet, 6 seulement modifient un indéfini. Cela va intuitivement dans le sens de la valeur d'introduction d'information nouvelle de la présentative, la clivée en c'est étant réputée reprendre une information partagée. La clivée en il y a semble être un schéma constructionnel privilégié pour résoudre la difficulté, décrite par Cappeau et Deulofeu, à accepter des indéfinis en sujet antéposé en raison du conflit entre information nouvelle et information ancienne supposée par le sujet, puisqu'elle permet de placer un «sujet sémantique » consistant en de l'information nouvelle en position syntaxique d'objet tout en formulant la prédication sous la forme d'une subordonnée introduite par un relatif sujet co-référent au sujet sémantique.

Nos analyses vont dans le sens de Michaelis et Duffield pour certains de leurs résultats : l’antécédent du relatif sujet qui est effectivement plus fréquemment objet direct dans la proposition dominante (278 occurrences soit 49,5\% des cas). En outre, le relatif sujet est plus à même de modifier un antécédent SN indéfini (254 occurrences sur 563) que le relatif objet par exemple (quelques cas isolés sur 244). Des analyses plus précises permettent d'affiner ces résultats. En effet, on observe des tendances différentes selon la fonction de l'antécédent dans la principale. Ainsi, les SN ou pronoms modifiés, quand ils sont objets directs, sont indéfinis dans 63,5\% des cas. En revanche pour les antécédents en position d'attribut, la tendance est inverse, et l'on ne dénombre plus que 28,5\% de SN ou pronoms indéfinis. Par ailleurs, il n’y a pas contrairement aux données de Michaelis et Duffield (2015) de répartition entre modification du sujet (assurée par le relatif objet objet à 60\% dans leur corpus) et modification du complément (assurée majoritairement par le relatif sujet dans leur corpus) : seul qui se rapporte à un antécédent sujet, et de façon quasi anecdotique (à 4 \% des occurrences en incluant les dislocations en rapport avec un clitique exerçant cette fonction). L'emploi massif du clitique sujet en français (Culberston 2010), et l'incapacité de le modifier par une relative semblent expliquer cette divergence entre français et anglais. 
L’analyse proposée soutient la hiérarchie d’accessibilité de Keenan et Comrie (1977). Plus il est accessible, plus un relatif est fréquent et moins il est sujet à l'erreur de performance. L'attente qu'un relatif moins accessible entre plus régulièrement dans des schémas collocatifs reste cependant à attester en comparant plus systématiquement que nous n'avons pu le faire des schémas de même type (constructionnels, type d’antécédent, lexicaux avec le verbe subordonné). Des évidences sont fournies de convergences entre statut informationnel et emplois de relatifs.

\section{Conclusion}

Malgré le caractère restreint du corpus utilisé, ces résultats montrent que les relatifs simples sont plus nombreux et moins sujets à l'erreur que les complexes. Ce travail ne permet cependant pas de départager une explication en terme de traitement, d'une autre en terme de construction, même si rien dans les données ne favorise fortement cette dernière. Une perspective d'application de ce travail est clairement la didactique, en particulier l'apprentissage sur corpus de formes fréquentes et schémas productifs du français: si le français vernaculaire a un ensemble de relatives différentes, l'apprentissage des usages normés en décalage avec le vernaculaire devrait poser des difficultés qu'il s’agit de documenter et de résoudre.

Nous avons pu documenter pour ce que nous appelons le français vernaculaire un ensemble de relatives différentes dans un échantillon de cadres interactionnels de plus ou moins grande proximité communicative (interactions peu ou pas préparées, familiarité des locuteurs plus ou moins grande, distance sociale et formalité du cadre, etc.). Notre échantillon assez restreint mériterait d'être élargi à d'autres types d'interaction et d'autres genres communicationnels, de l'écrit notamment, pour examiner une probable variation dans les usages des relatifs, qu'elle soit qualitative ou quantitative. Une meilleure connaissance de cette variation permettrait notamment un meilleur profilage linguistique en fonction des objectifs communicatifs des apprenants.

\section{Références bibliographiques}

Alexiadou, Artemis, Paul Law, Andre Meinunger et Chris Wilder (dirs). (2000). The Syntax of Relative Clauses. Amsterdam : Benjamins.

Auger, Julie. (1995). On the history of relative clauses in French and some of its dialects. H. Andersen (dir.). Historical Linguistics 1993. Amsterdam : Benjamins.

Baude, Olivier et E. Guerin. (à paraitre). « Pourquoi et comment dresser le portrait sonore d'une grande ville ? L’exemple d'ESLO 2 » dans F. Gadet (dir.), Actes du colloque Les métropoles francophones en temps de globalisation.

Behney, Jennifer et Susan Gass. (2013). Accessibility Hierarchy. A study using syntactic priming. John W. Schwieter (dir.). Innovative research and practices in Second Language Acquisition and bilingualism. Amsterdam : Benjamins. 43-61.

Benzitoun, Christophe, Karen Fort et Benoit Sagot. (2012). TCOF-POS : un corpus libre de français parlé annoté en morphosyntaxe. Actes de la conférence conjointe JEP-TALN-RECITAL, Grenoble, 4 au 8 juin 2012. Volume 2 : TALN. 99-112.

Blanche-Benveniste, Claire et Philippe Martin. (2010). Le français. Usages de la langue parlée. Leuven et Paris : Peeters. 
Blanche-Benveniste, Claire. (1990). Usages normatifs et non normatifs dans les relatives en français, en espagnol et en portugais. Johannes Bechert, Giuliano Bernini et Claude Buridant (dirs). Towards a Typology of European Languages. Berlin : de Gruyter. 317-225.

Blanche-Benveniste (2000) : Approches de la langue parlée en français. Paris : Ophrys.

Blanche-Benveniste, Claire. 1980. Divers types de relatives en français parlé. TA Informations Grenoble 21,2, 16-25.

Cappeau, Paul et José Deulofeu. (2001). Partition et topicalisation : il y a “stabilisateur" de sujets et de topiques indéfinis. Cahiers de Praxématique 37, 45-82.

Collins, Peter. 1991. " Pseudo-clefts end clefts constructions: a thematic and informatical Interpretation ». Linguistics 29,3, 481-519.

Comrie, Bernard et Tania Kuteva. (2005). Relativization strategies. Martin Haspelmath, Matthew Dryer, David Gil et Bernard Comrie (dirs). The World Atlas of Language Structures. Oxford : Oxford University Press. 494-501.

Culbertson, Jenny. (2010). Convergent evidence for categorial change in French: from subject clitic to agreement marker. Language 86,1, 85-132.

Diessel, Holger. 2007. The emergence of relative clauses in early child language. MS, Universität Jena.

Diessel, Holger et M. Tomasello. (2000). The development of relative clauses in spontaneous child speech. Cognitive Linguistics 11, 131-151.

Eshkol-Taravella, I., Olivier Baude, D. Maurel, L. Hriba, C. Dugua, I. Tellier. (2012). Un grand corpus oral « disponible » : le corpus d’Orléans 1968-2012. TAL 52,3, 17-46.

ESLO_V1 : Enquêtes Sociolinguistiques à Orléans, 2013, LLL-UMR 7270, Université d’Orléans, http://eslo.huma-num.fr

Gadet, Françoise. (1995). Les relatives non standard en français parlé, le système et l'usage. Études romanes 34, 141-162.

Gadet, Françoise. (1989). La relative non standard saisie par les grammaires. Linx 20, 37-49.

Gapany, Joël. (2004). Formes et fonctions des relatives en français: étude syntaxique et sémantique. Berne : Peter Lang.

Godard, Danièle. (1988). La syntaxe des relatives en français. Paris : CNRS.

Guasti, Maria Teresa, Stavroula Stavrakaki et Fabrizio Arosio. (2012). Cross-linguistic differences and similarities in the acquisition of relative clauses: Evidence from Greek and Italian. Lingua 122,6, 700713.

Guasti, Maria Teresa et Ur Shlonsky. (1995). The acquisition of French relative clauses reconsidered. Language Acquisition 4,4, 257-276.

Guiraud, Pierre. (1966). Les relatives non standard en français parlé, le système et l'usage. Langages 1,3, 40-48.

Haspelmath, Martin. (2010). Comparative concepts and descriptive categories in crosslinguistic studies. Language 86,3, 663-687. 
Hawkins, Roger. (1989). The acquisition of French subject, direct object and genitive restrictive relative clauses by second language learners. Second Language Research December 5,2, 156-188.

Hendery, Rachel. (2012). Relative clauses in time and space. Amsterdam : Benjamins.

Keenan, Edward L. et Bernard Comrie. (1977). Noun Phrase Accessibility and Universal Grammar. Linguistic Inquiry 8,1, 63-99.

Lambrecht, Knud (2001). A framework for the analysis of cleft constructions. Linguistics 39, 3. 463-516.

Kidd, Evan (dir.). (2011). The acquisition of relative clauses. Processing, typology and function. Amsterdam : Benjamins.

Lambrecht, Knud. 1987. « Sentence focus, information structure, and the thetic categorical distinction ». J. Aske, N. Beery, L. Michaelis et H. Filip (dirs). Proceedings of the 13th Annual Meeting of the Berkeley Linguistics Society, February 14-16, 1987, General Session and Parasession on Grammar and Cognition. 366-382.

Lambrecht, Knud 1988. Presentational cleft constructions in spoken French". In J. Haiman and S. Thompson (dirs) Clause combining in grammar and discourse. Amsterdam: John Benjamins. 135179.

Lambrecht, Knud 2001. «A framework for the analysis of cleft constructions.» Linguistics 39, 3. 463516.

Lefebvre, Claire et Robert Fournier. (1978). Les relatives en français de Montréal. Cahier de linguistique 8, 273-294.

Lin, Yow-Yu. (2015). Developmental trajectory of relative clauses among Mandarin learners of English. Concentric: Studies in Linguistics 41, 1, 63-94.

Matras, Yaron et Hans-Jürgen Sasse (dirs). 1995, Verb-subject order and theticity in European languages, Sprachtypologie und Universalienforschung. 48, 1/2.

Meisel, Jürgen, Martin Elsig et Matthias Bonnesen. (2011). Delayed grammatical acquisition in first language development: Subject-verb inversion and subject clitics in French interrogatives. Linguistic Approaches to Bilingualism 1,4, 347-390.

Méry, Renault. (2011). L’idée de thématisation et le concept de repérage. In Jean-Marie Merle et Charles Zaremba (dir.), Organisation informative et structuration de l'énoncé. Les prépositions, Travaux du Claix, 21, Presses universitaires de Provence.

Morel M.-A. (1992) : « Les présentatifs en français », in La deixis, Paris, PUF : 507-517.

Mirault, Hugues. (1997). La syntaxe des propositions relatives. Berne : Peter Lang.

Murelli, Adriano. (2011). Relative Constructions in European Non-Standard Varieties. Berlin : de Gruyter.

Nølke, Henning. 1983. « Quelques réflexions sur la structure sémantique des phrases clivées en français moderne ». Modèles linguistiques V,1, 117-140.

Pierrard, Michel. (1988). La relative sans antécédent en français moderne: essai de syntaxe propostionnelle. Paris : Peeters. 
Scappini, Sophie-Anne. 2006. Étude du dispositif en c'est...qu, différenciation entre une relative en c'est...qu et une proposition clivée. Thèse de doctorat, Université de Provence. 